PROCEEDINGS OF THE

AMERICAN MATHEMATICAL SOCIETY

Volume 137, Number 10, October 2009, Pages 3169-3176

S 0002-9939(09)10013-8

Article electronically published on June 3, 2009

\title{
CONSTRUCTION OF COVERS IN POSITIVE CHARACTERISTIC VIA DEGENERATION
}

\author{
IRENE I. BOUW
}

(Communicated by Ted Chinburg)

\begin{abstract}
In this paper we construct examples of covers of the projective line in positive characteristic such that every degeneration is inseparable. The result illustrates that it is not possible to construct all covers of the generic $r$-pointed curve of genus zero inductively from covers with a smaller number of branch points.
\end{abstract}

Let $k$ be an algebraically closed field of characteristic $p>0$. Let $X=\mathbb{P}_{k}^{1}$ and $G$ be a finite group. We fix $r \geq 3$ distinct points $\mathbf{x}=\left(x_{1}, x_{2}, \ldots, x_{r}\right)$ on $X$. We ask whether there exists a tame Galois cover $\pi: Y \rightarrow X$ with Galois group $G$ which is branched at the $x_{i}$. If $p$ does not divide the order of $G$, then the answer is well known. Namely, such a cover exists if and only if $G$ may be generated by $r-1$ elements.

Suppose that $p$ divides the order of $G$. Then the existence of a $G$-cover as above depends on the position of the branch points $x_{i}$ (see, for example, [8, Lemma 6]). In this paper we restrict to the case that $(X ; \mathbf{x})$ is the generic $r$-pointed curve of genus zero. A more precise version of the existence question in positive characteristic is whether there exists a $G$-Galois cover of $(X ; \mathbf{x})$ with given ramification type (see, for example, 8$]$ ). For the particular kinds of groups we consider here, we define the ramification type in 81 .

Osserman ([5]) proves (non)existence of covers in positive characteristic, for certain ramification types. His method is roughly as follows. First, he proves results for covers branched at $r=3$ points. In this case his results are strongest. Using the case $r=3$, he then constructs admissible covers of degenerate curves which deform to covers of smooth curves (see $₫ 2$ for a definition).

Suppose we are given a tame $G$-Galois cover $\pi$ of $\left(X=\mathbb{P}_{k}^{1} ; \mathbf{x}\right)$. Osserman $([\underline{5}, \S 6])$ asks whether there exists a degeneration $(\bar{X}, \overline{\mathbf{x}})$ of $(X ; \mathbf{x})$ such that $\pi$ specializes to an admissible cover of $(\bar{X}, \overline{\mathbf{x}})$. If such a degeneration exists, he says that $\pi$ has a good degeneration. Covers which admit a good degeneration are exactly those which may be shown to exist inductively from the existence of covers with fewer branch points. The goal of this paper is to produce covers which do not have a good degeneration. We show that such covers exist with an arbitrarily large number of branch points.

Received by the editors September 13, 2007.

2000 Mathematics Subject Classification. Primary 14H30, 14H10.

(C)2009 American Mathematical Society Reverts to public domain 28 years from publication 
The covers of $X=\mathbb{P}_{k}^{1}$ we consider have Galois group $G=(\mathbb{Z} / p \mathbb{Z})^{n} \rtimes \mathbb{Z} / m \mathbb{Z}$ and ramification of order prime to $p$. The existence of such covers can be expressed in terms of (a generalization of) the $p$-rank of the corresponding $m$-cyclic cover $Z \rightarrow X([2])$. We find covers without a good degeneration by comparing the $p$-rank of $Z$ with the $p$-rank of its degenerations.

I thank Brian Osserman for suggesting that I write this paper and for helpful remarks.

\section{Meta-ABelian COVERS}

In this section we recall a result from 2 on the existence of tame Galois covers with Galois group $G \simeq(\mathbb{Z} / p)^{n} \rtimes \mathbb{Z} / m$ in characteristic $p>0$. Let $p \neq 2$ be a prime and $m$ be an integer prime to $p$. We suppose that $k$ is an algebraically closed field of characteristic $p$.

Let $\mathbf{x}=\left(x_{1}, \ldots, x_{r}\right)$ be $r$ distinct $k$-rational points of $X \simeq \mathbb{P}_{k}^{1}$. Let $\mathbf{a}=$ $\left(a_{1}, \ldots, a_{r}\right)$ be an $r$-tuple of integers with $0<a_{i}<m$ and $\sum a_{i} \equiv 0 \bmod m$. Suppose moreover that $\operatorname{gcd}\left(m, a_{1}, \ldots, a_{r}\right)=1$. Let $g: Z \rightarrow X$ be the $m$-cyclic cover of type $(\mathbf{x} ; \mathbf{a})(2])$; i.e. $Z$ is the complete nonsingular curve obtained by completing and normalizing the curve given by the equation

$$
z^{m}=\prod_{i: x_{i} \neq \infty}\left(x-x_{i}\right)^{a_{i}}
$$

and $g:(x, z) \mapsto x$.

We may extend the definition of the type also to disconnected covers. Let $\mathbf{a}=$ $\left(a_{1}, \ldots, a_{r}\right)$ be an $r$-tuple of integers with $0<a_{i}<m$ and $\sum a_{i} \equiv 0 \bmod m$, and let $n=\operatorname{gcd}\left(m, a_{1}, \ldots, a_{r}\right)$. Define a (disconnected) $m$-cyclic cover $g: Z \rightarrow \mathbb{P}^{1}$, as follows. Let $Z^{\prime} \rightarrow \mathbb{P}^{1}$ be the $(m / n)$-cyclic cover of type $\left(a_{1} / n, \ldots, a_{r} / n\right)$, and let $Z=\operatorname{Ind}_{\mathbb{Z} /(m / n) \mathbb{Z}}^{\mathbb{Z} / m \mathbb{Z}} Z^{\prime}$ be the induced curve; i.e. $Z$ consists of $n$ disjoint copies of $Z^{\prime}$ which are indexed by the cosets of $\mathbb{Z} /(m / n) \mathbb{Z}$ in $\mathbb{Z} / m \mathbb{Z}$.

From now on, we suppose again that $Z$ is connected. We denote by $\sigma(Z)$ the $p$ rank of $Z$. Then $\sigma(Z)=\operatorname{dim}_{\mathbb{F}_{p}} V$, where $V:=\operatorname{Hom}\left(\pi_{1}(Z), \mathbb{Z} / p\right)$. Since $\mathbb{Z} / m \mathbb{Z}$ acts on $V$, there exists a tame $G:=V \rtimes \mathbb{Z} / m \mathbb{Z}$-Galois cover $\pi: Y \rightarrow X$ which factors through $Z$. We sometimes call this cover, somewhat imprecisely, the maximal metaabelian cover corresponding to $g: Z \rightarrow X$.

Proposition 1.1 gives an upper bound on $\sigma(Z)$ which is attained if the branch points $x_{i}$ are sufficiently general. For a more precise version, we refer to [2]. To formulate this upper bound, we need to introduce some notation.

Let $f$ be the order of $p$ in $\mathbb{Z} / m \mathbb{Z}^{*}$ and let $\zeta_{m} \in \mathbb{F}_{p^{f}}$ be a primitive $m$ th root of unity. For every $i \in\{1, \ldots, m-1\}$ we let $\chi_{i}: \mathbb{Z} / m \mathbb{Z} \rightarrow \mathbb{F}_{p^{f}}^{\times}$with $\chi_{i}(1)=\zeta_{m}^{i}$ be the character of a nontrivial, irreducible representation of $\mathbb{Z} / m \mathbb{Z}$. Let $I=\{1, \ldots, m-$ 1\}/ $\sim$, where $i \sim p^{j} i$. Then $I$ corresponds to the set of nontrivial, irreducible characters $\mathbb{Z} / m \mathbb{Z} \rightarrow \mathbb{F}_{p}^{\times}$. Namely, for every $i \in I$ the character $\chi_{i}+\chi_{p i}+\cdots+\chi_{p^{n_{i}-1}} i$ is a nontrivial, irreducible $\mathbb{F}_{p}$-character. Here $n_{i}$ is the number of elements of the equivalence class of $i$; i.e. $0<n_{i} \leq f$ is minimal with the property that $i \equiv p^{n_{i}} i$ $(\bmod m)$. 
If $a$ is an integer, we denote by $\langle a\rangle$ the unique integer with $\langle a\rangle \equiv a(\bmod m)$ and $0 \leq\langle a\rangle<m$. For $i \in\{1, \ldots, m-1\}$ we define

$$
\gamma(i)=\frac{1}{m}\left(\sum_{j=1}^{r}\left\langle i a_{j}\right\rangle\right) .
$$

Then $\gamma(i)-1$ is the dimension of the $\chi_{i}$ th eigenspace of $H^{1}\left(Z, \mathcal{O}_{Z}\right)$. It is well known that this number is an upper bound for the dimension of the $\chi_{-i}$ th eigenspace of $V \otimes_{\mathbb{F}_{p}} \mathbb{F}_{p^{f}}$. This observation is used in [2] to conclude that

$$
B(\mathbf{a}):=\sum_{i \in I} n_{i} \min _{0 \leq t \leq f-1}\left(\gamma\left(p^{t} i\right)-1\right)
$$

is an upper bound on the $p$-rank of $Z$. Since $\chi_{i}+\chi_{p i}+\cdots+\chi_{p^{n_{i}-1} i}$ is a nontrivial,

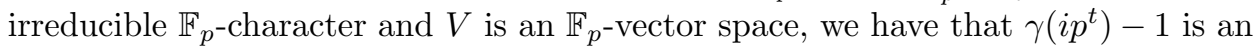
upper bound for the dimension of the $\chi_{-i}$ th eigenspace of $V \otimes_{\mathbb{F}_{p}} \mathbb{F}_{p^{f}}$ for every $t$. This explains why we take the minimum in the formula for $B(\mathbf{a})$ above.

We recall the result.

Proposition 1.1. (a) We have that

$$
\sigma(Z) \leq B(\mathbf{a})
$$

(b) Suppose that $p \geq m(r-3)$. There exists $x_{1}, \ldots, x_{r} \in X=\mathbb{P}_{k}^{1}$ such that

$$
\sigma(Z)=B(\mathbf{a}) \text {. }
$$

Proof. Let $V_{i}$ be the $\chi_{-i}$ th eigenspace of $V \otimes_{\mathbb{F}_{p}} \mathbb{F}_{p^{f}}$. In [2, Lemma 4.3] it is shown that $\operatorname{dim}_{\mathbb{F}_{p}} V_{i} \leq \gamma(i)-1$. The upper bound on $\sigma(Z)$ now follows by summing over all character spaces (2, (1)]). Part (b) follows from [2, Theorem 6.1].

In 2] one finds some variants of this result: under certain additional hypotheses on the type, we may weaken the condition on $p$. (Compare to Remark 2.5.) In this paper, we use the following special case of Proposition 1.1

Corollary 1.2. Let $g: Z \rightarrow X$ be an $m$-cyclic cover of type $(\mathbf{x} ; \mathbf{a})$, where $(X ; \mathbf{x})$ is generic. Suppose that $p \geq m(r-3)$. The curve $Z$ is ordinary if and only if $\gamma(i)=\gamma\left(p^{s} i\right)$, for all $i$ and $s$.

Proof. Proposition 1.1.(b) implies that the $p$-rank $\sigma(Z)$ of $Z$ equals $B(\mathbf{a})$. Since $\gamma(i)-1$ is the dimension of the $\chi_{i}$ th eigenspace of $H^{1}(Z, \mathcal{O})$, it follows that $B(\mathbf{a})=$ $g(Z)=\operatorname{dim}_{k} H^{1}(Z, \mathcal{O})$ if and only if $\gamma(i)=\gamma\left(p^{s} i\right)$ for all $i$ and $s$. The corollary follows.

The bound $p \geq m(r-3)$ in Corollary 1.2 is probably not optimal. However, it follows from the main result of $[3$ that one cannot omit it in general.

\section{Degeneration}

Let $G$ be a finite group with trivial center, and let $r \geq 3$ be an integer. We denote by $\mathcal{H}_{r, G}$ the stack over $\operatorname{Spec}(\mathbb{Z})$ which parameterizes tame $G$-Galois covers of a curve $X$ of genus zero with $r$ ordered branch points ([6]). We may choose an isomorphism $X \simeq \mathbb{P}^{1}$ which sends $\left(x_{1}, x_{2}, x_{3}\right)$ to $(0,1, \infty)$.

There exists a stack $\mathcal{H}_{r, G}^{\text {adm }}$ of admissible $G$-Galois covers, which contains $\mathcal{H}_{r, G}$ as a dense open substack ([6, Section 5]). An admissible cover is a certain type 
of degenerate cover between semistable curves. The stack $\mathcal{H}_{r, G}^{\text {adm }}$ is complete over $\operatorname{Spec}(\mathbb{Z}[1 /|G|])$. We refer to $[8, \S 2.1]$ for a short introduction to admissible covers. The natural map $\mathcal{H}_{r, G} \rightarrow \mathcal{M}_{0, r}$, which sends a tame $G$-Galois cover with branch points $\mathbf{x}=\left(x_{1}, \ldots, x_{r}\right)$ to the pointed curve $(X ; \mathbf{x})$, extends to a map $\mathcal{H}_{r, G}^{\text {adm }} \rightarrow \overline{\mathcal{M}}_{0, r}$. Here $\overline{\mathcal{M}}_{0, r}$ is the stack of stably marked curves of genus zero.

For our purposes, it will be enough to consider $R$-valued points, where $R$ is a discrete valuation ring.

Definition 2.1. Let $\pi_{K}: Y_{K} \rightarrow X_{K}$ be a $K$-valued point of $\mathcal{H}_{r, G}$. We say that $\pi_{K}$ has a good degeneration if there exists a finite extension $L / K$ and a discrete valuation ring $R \subset L$ such that $\pi_{K} \otimes_{K} L$ extends to an $R$-valued point of $\mathcal{H}_{r, G}^{\text {adm }}$.

If $\pi: \mathcal{Y} \rightarrow \mathcal{X}$ is an admissible $G$-cover over $\operatorname{Spec}(R)$ with $\pi \otimes_{R} L \simeq \pi_{K} \otimes_{K} L$, we call $\pi: \mathcal{Y} \rightarrow \mathcal{X}$ (or also its special fiber $\bar{\pi}:=\pi \otimes_{R} k: \bar{Y} \rightarrow \bar{X}$ ) a good degeneration of $\pi_{K}$. Here $k$ is the residue field of $R$.

In the rest of this paper, we assume that $K$ is a field of characteristic $p>0$. An $r$-pointed curve $\left(X_{K} ; \mathbf{x}\right)$ is called generic if $\left(X_{K} ; \mathbf{x}\right)$ corresponds to the generic point of the moduli space $\mathcal{M}_{0, r} \otimes \mathbb{F}_{p}$ of $r$-pointed curves in characteristic $p$.

Lemma 2.2. Let $\left(X_{K} ; \mathbf{x}\right)$ be generic. Let $\pi_{K}: Y_{K} \rightarrow X_{K}$ be a tame $G$-Galois cover which has a good degeneration. Then there exists a good degeneration $\bar{\pi}: \bar{Y} \rightarrow \bar{X}$ of $\pi_{K}$ such that the special fiber $\bar{X}$ of $\mathcal{X}$ consists of two components $X_{1}, X_{2}$ meeting in one point $\tau$.

Proof. The irreducible components of $\mathcal{H}_{r, G}^{\mathrm{adm}} \backslash \mathcal{H}_{r, G}$ have codimension 1 in $\mathcal{H}_{r, g}^{\mathrm{adm}}$. Moreover, the generic point of such a component maps to the generic point of an irreducible component of the boundary of $\overline{\mathcal{M}}_{0, r}$. The generic point of such an irreducible component corresponds to a semistable, marked curve of genus zero consisting of two projective lines intersecting in one point.

We now consider the special case of meta-abelian covers, as in 91 . We want to construct $m$-cyclic covers $g_{K}$ such that the corresponding maximal meta-abelian cover $\pi_{K}$ does not have a good degeneration. Let $g_{K}: Z_{K} \rightarrow X_{K}$ be an $m$-cyclic cover and $\pi_{K}: Y_{K} \rightarrow X_{K}$ the corresponding maximal meta-abelian cover. Then $\pi_{K}$ has a good degeneration if and only if there exists an admissible degeneration $\bar{g}: \bar{Z} \rightarrow \bar{X}$ of $g_{K}: Z_{K} \rightarrow X_{K}$ with $\sigma(\bar{Z})=\sigma\left(Z_{K}\right)$. The $p$-rank of a semistable curve $\bar{Z}$ is defined as $\operatorname{dim}_{\mathbb{F}_{p}} H^{1}(\bar{Z}, \mathcal{O})^{F}$, where $F$ denotes the absolute Frobenius morphism ([1, $\S 1])$. It is well known that $\sigma(\bar{Z})$ is the sum of the $p$-rank of its irreducible components and the Betti number of the dual graph of $\bar{Z}$ ([1, Lemma 1.3]). In particular, $\bar{Z}$ is ordinary if and only if all its irreducible components are ordinary. Lemma 2.2 implies that it suffices to consider degenerations of $\pi_{K}$, where $X_{K}$ degenerates into two projective lines.

The following lemma describes the type of the restriction of $\bar{g}$ to $X_{i}$. Together with Proposition [1.1, we obtain a bound on $\sigma(\bar{Z})$ which may be used to show that a given degeneration is a bad degeneration. See Example 2.4 for an easy example.

Lemma 2.3. Let $g_{K}: Z_{K} \rightarrow X_{K}$ be an $m$-cyclic cover of type $(\mathbf{x} ; \mathbf{a})$. Let $\bar{g}: \bar{Z} \rightarrow \bar{X}$ be a degeneration of $g_{K}$ such that $\bar{X}$ consists of two irreducible components $X_{1}, X_{2}$ intersecting in one point $\tau$. Write $\bar{g}_{i}: Z_{i} \rightarrow X_{i}$ for the restriction of $\bar{g}$ to $X_{i}$. Let $S_{i} \subset\{1, \ldots, r\}$ be the subset of indices $j$ such that $x_{j}$ specializes to $X_{i}$. Then $g_{i}$ 
has type

$$
\left(\left(x_{j}\right)_{j \in S_{i}} \cup(\tau) ;\left(a_{j}\right)_{j \in S_{i}} \cup\left(\sum_{j \notin S_{i}} a_{j}\right)\right) .
$$

Proof. This follows immediately from the definition of the type. The curve $Z_{i}$ need not be connected, but this is not a problem.

Before constructing covers with do not admit a good degeneration, we recall how to construct examples of covers which do have a good degeneration.

A well-known result of formal patching (4, 7]) states that every tame admissible cover may be deformed to a cover of smooth curves. Let $\bar{g}: \bar{Z} \rightarrow \bar{X}$ be an admissible cover which deforms to a smooth $m$-cyclic cover $g_{K}: Z_{K} \rightarrow X_{K}$ of type a. Suppose that $\sigma(\bar{Z})=B(\mathbf{a})$. Since $\sigma\left(Z_{K}\right) \geq \sigma(\bar{Z})=B(\mathbf{a})$ it follows that $\sigma(Z)=\sigma(\bar{Z})$. Therefore the maximal meta-abelian cover corresponding to $\pi_{K}$ has a good degeneration in this case. Examples of this situation can be found in [1, Section 4].

We give an easy example of a cover which does not have a good degeneration.

Example 2.4. Let $m=5$ and let $p \equiv-1(\bmod m)$. Then the order $f$ of $p$ in $\mathbb{Z} / m \mathbb{Z}^{*}$ is 2 . We consider $\mathbf{a}=(1,1,1,2)$. One computes that $B(\mathbf{a})=2$. Proposition 1.1 implies that for $p$ sufficiently large there exists a tame $G=(\mathbb{Z} / p \mathbb{Z})^{2} \rtimes \mathbb{Z} / m \mathbb{Z}$ Galois cover $\pi_{K}: Y_{K} \rightarrow \mathbb{P}_{K}^{1}$ branched at 4 points which factors through a cover of type a. In fact, 2, Proposition 7.8] implies that we do not need the lower bound on $p$ in this case.

Let $\bar{\pi}: \bar{Y} \rightarrow \bar{X}$ be a degeneration of $\pi_{K}$ and let $\bar{g}: \bar{Z} \rightarrow \bar{X}$ be the degeneration of the corresponding $m$-cyclic cover. Then $\bar{X}$ consists of 2 irreducible components, which we denote by $X_{1}$ and $X_{2}$. To each of these components specialize two of the points $x_{1}, \ldots, x_{4}$. Lemma 2.3 implies that (up to renumbering) the restrictions $g_{1}: Z_{1} \rightarrow X_{1}$ and $g_{2}: Z_{2} \rightarrow X_{2}$ of $\bar{g}$ have type $\mathbf{a}_{1}=(1,1,3)$ and $\mathbf{a}_{2}=(2,1,2)$. One computes that $B\left(\mathbf{a}_{i}\right)=0$ for $i=1,2$. This implies that $\sigma\left(Z_{1}\right)=\sigma\left(Z_{2}\right)=0$. Since $\bar{g}$ is totally branched over the singular point, there are no loops. Therefore $\sigma(\bar{Z})=0<2=\sigma\left(Z_{K}\right)=B(\mathbf{a})$ for every admissible degeneration of $g_{K}$. We conclude that $\pi_{K}$ does not have a good degeneration.

Remark 2.5. Suppose that $p \equiv 1(\bmod m)$ and let $\left(X=\mathbb{P}_{K}^{1} ; \mathbf{x}\right)$ be the generic $r$-pointed curve of genus zero. Let $g_{K}: Z_{K} \rightarrow X_{K}$ be an $m$-cyclic cover of $X_{K}$ branched at the $x_{i}$. Then it is shown in [2, Proposition 7.4] that $g_{K}$ has an ordinary degeneration $\bar{g}$. Therefore the maximal meta-abelian cover of $X_{K}$ corresponding to $g_{K}$ has a good degeneration.

In the case that $p \equiv-1(\bmod m)$ it is shown in [2, Proposition 7.8] that every $m$-cyclic cover of $\left(X=\mathbb{P}_{k}^{1} ; \mathbf{x}\right)$ has a good degeneration, provided that the number of branch points is at least 5 . The result of $₫ 3$ suggests that a similar statement does not hold for $f \geq 3$.

\section{Covers without a Good Degeneration}

In this section, we produce a family of examples of Galois covers with an arbitrarily large number of branch points which do not have a good degeneration. Let $f$ be an odd prime and put $\alpha:=2$. Define $m:=\alpha^{f}-1=1+\alpha+\cdots+\alpha^{f-1}$. We define

$$
\mathbf{a}=\left(1, \alpha, \alpha^{2}, \ldots, \alpha^{f-1}\right) .
$$


We suppose that $\left(X=\mathbb{P}_{K}^{1} ; \mathbf{x}\right)$ is the generic $f$-pointed curve of genus zero in characteristic $p$ and let $g_{K}: Z_{K} \rightarrow X_{K}=\mathbb{P}_{K}^{1}$ be the $m$-cyclic cover of type $(\mathbf{x} ; \mathbf{a})$.

As in $₫ 1$, we define $\gamma(i)=\left(\sum_{t=0}^{f-1}\left\langle i \alpha^{t}\right\rangle\right) / m$ for every $i \in\{1, \ldots, m-1\}$. Since we have chosen $\alpha=2$, every such $i$ may be written as $i=\sum_{j \in S} \alpha^{j}$ for some $S \subsetneq\{0, \ldots, f-1\}$.

Lemma 3.1. Let $S \subsetneq\{0, \ldots, f-1\}$ and $i:=\sum_{j \in S} \alpha^{j}$. Then

$$
\gamma(i)=|S|, \quad \gamma(m-i)=f-|S| .
$$

Proof. Let $i$ be as in the statement of the lemma. The definition of $m$ implies that $\alpha^{f} \equiv 1(\bmod m)$. Therefore

$$
\left\langle i \alpha^{t}\right\rangle=\sum_{j \in S}\left\langle\alpha^{t+j}\right\rangle
$$

This implies that

$$
\gamma(i)=\frac{1}{m} \sum_{t=0}^{f-1} \sum_{j \in S}\left\langle\alpha^{j+t}\right\rangle=\frac{1}{m} \sum_{j \in S}\left(1+\alpha+\cdots+\alpha^{f-1}\right)=|S| .
$$

The second statement follows immediately from the first statement and the definition of $m$.

Lemma 3.2. Suppose that $p=\operatorname{char}(K)$ satisfies $p \geq m(f-3)$ and $p \equiv \alpha^{i}(\bmod m)$ for some $i \not \equiv 0(\bmod f)$. Let $g_{K}: Z_{K} \rightarrow \mathbb{P}_{K}^{1}$ be an $m$-cyclic cover of type $(\mathbf{x} ; \mathbf{a})$, where $\mathbf{a}$ is as in (11) and $\left(\mathbb{P}^{1} ; \mathbf{x}\right)$ is the generic $r$-pointed curve. Then $Z_{K}$ is ordinary, i.e.

$$
b:=B(\mathbf{a})=g\left(Z_{K}\right)=(f-1)(m-1) / 2 .
$$

In particular, there exists a tame $G:=(\mathbb{Z} / p \mathbb{Z})^{b} \rtimes \mathbb{Z} / m \mathbb{Z}$-Galois cover $\pi_{K}: Y_{K} \rightarrow \mathbb{P}_{K}^{1}$ of type $\mathbf{a}$.

Proof. The assumption $p \equiv \alpha^{i}$ implies that $\gamma\left(s p^{j}\right)=\gamma(s)$, for all $j$ and $s$. The statement now follows immediately from Corollary 1.2 .

From now on, we let $p$ be as in Lemma 3.2. Note that $p$ has order $f$ in $\mathbb{Z} / m \mathbb{Z}^{*}$, since $f$ is prime. The goal of this section is to show that the maximal meta-abelian cover $\pi_{K}$ corresponding to $g_{K}$ does not have a good degeneration. It suffices to show that every degeneration $\bar{g}: \bar{Z} \rightarrow \bar{X}$ of $g_{K}$ is nonordinary. Lemma 2.2 implies that it suffices to consider degenerations $\bar{g}: \bar{Z} \rightarrow \bar{X}$ of $g_{K}: Z_{K} \rightarrow X_{K}$ such that $\bar{X}$ consists of two irreducible components $X_{1}$ and $X_{2}$ intersecting in one point $\tau$.

Consider such a degeneration $\bar{g}: \bar{Z} \rightarrow \bar{X}$. We let $S_{i} \subset\{0, \ldots, f-1\}$ be the set of indices $j$ such that $x_{j}$ specializes to $X_{i}(i=1,2)$. We may assume that $2 \leq\left|S_{1}\right| \leq\left|S_{2}\right| \leq f-2$, for $i=1,2$. In particular, $\left|S_{1}\right| \leq(f-1) / 2$.

Proposition 3.3. Let $\bar{g}: \bar{Z} \rightarrow \bar{X}$ be a degeneration of $g_{K}: Z_{K} \rightarrow X_{K}$. Then $\bar{Z}$ is nonordinary.

Proof. We write $g_{i}: Z_{i} \rightarrow X_{i}$ for the restriction of $\bar{g}$ to $X_{i}$. The curve $\bar{Z}$ is ordinary if and only if $Z_{i}$ is ordinary for $i=1,2$ (see the discussion before Lemma 2.3).

We define

$$
\gamma_{1}(i)=\frac{1}{m}\left(\left\langle\sum_{j \notin S_{1}} i \alpha^{j}\right\rangle+\sum_{j \in S_{1}}\left\langle i \alpha^{j}\right\rangle\right) .
$$


These are the terms occurring in the bound for the cover $g_{1}: Z_{1} \rightarrow X_{1}$ (Lemma2.3). Note that $g_{1}$ is branched at $\left|S_{1}\right|+1$ points, namely the specialization of $x_{j}$ for $j \in S_{1}$ and the singular point $\tau$. It follows from Corollary 1.2 that $Z_{1}$ is ordinary if and only if $\gamma_{1}(i)=\gamma_{1}\left(p^{t} i\right)$, for all $i$ and $t$.

Let $s=\sum_{j \in S_{1}} \alpha^{j}$. Choose an $0<\ell<f$ such that there exist $j_{1}, j_{2} \in S_{1}$ with $\ell \equiv j_{1}-j_{2}(\bmod f)$, and put $i=1+\alpha^{\ell}$. Such an $\ell$ exists since $\left|S_{1}\right| \geq 2$.

We claim that there exists a $t$ such that $\gamma_{1}\left(i \alpha^{t}\right) \neq \gamma_{1}(i)$. The proposition follows from this claim, since $p \equiv \alpha^{t}$ for some $t \not \equiv 0(\bmod f)$ and $f$ is a prime. To prove the claim, it suffices to show that $\sum_{t=0}^{f-1} \gamma_{1}\left(i \alpha^{t}\right) \not \equiv 0(\bmod f)$. Put

$$
d_{i}=m \gamma_{1}(i)-\sum_{j \in S_{1}}\left\langle i \alpha^{j}\right\rangle=\left\langle i \sum_{j \notin S_{1}} \alpha^{j}\right\rangle=\langle i(m-s)\rangle=\langle-i s\rangle .
$$

Here we used that $m=\sum_{j \notin S_{1}} \alpha^{j}+\sum_{j \in S_{1}} \alpha^{j}=\sum_{j \notin S_{1}} \alpha^{j}+s$.

We compute that

$$
\sum_{t=0}^{f-1} \gamma_{1}\left(i \alpha^{t}\right)=\frac{1}{m} \sum_{t=0}^{f-1} \sum_{j \in S_{1}}\left\langle i \alpha^{t+j}\right\rangle+\frac{1}{m} \sum_{t=0}^{f-1}\left\langle i \alpha^{t} \sum_{j \notin S_{1}} \alpha^{j}\right\rangle=\sum_{j \in S_{1}} \gamma\left(i \alpha^{j}\right)+\gamma\left(d_{i}\right) .
$$

Lemma 3.1 implies that $\gamma\left(i \alpha^{j}\right)=2$ for all $j$. Moreover,

$$
\gamma\left(d_{i}\right)=\gamma(\langle-i s\rangle)=f-\gamma(\langle i s\rangle) .
$$

Therefore

$$
\sum_{t=0}^{f-1} \gamma_{1}\left(i \alpha^{t}\right) \equiv 2\left|S_{1}\right|-\gamma(\langle i s\rangle) \quad(\bmod f) .
$$

We remark that $i s=\sum_{j \in S_{1}}\left\langle\alpha^{j}\right\rangle+\sum_{j \in S_{1}}\left\langle\alpha^{j+\ell}\right\rangle(\bmod m)$. We may write $\langle i s\rangle=$ $\sum_{j \in S}\left\langle\alpha^{j}\right\rangle$, for some $S \subset\{0, \ldots, f-1\}$. Since there exist $j_{1}, j_{2} \in S_{1}$ with $j_{1}-j_{2} \equiv \ell$ $(\bmod f)$ and $\alpha=2$, the cardinality of $S$ is strictly smaller than $2\left|S_{1}\right|$. Since we assumed that $\left|S_{1}\right| \leq(f-1) / 2$, it follows that $\gamma(i s) \not \equiv 2\left|S_{1}\right|(\bmod f)$. This implies that $\sum_{t=0}^{f-1} \gamma_{1}\left(i \alpha^{t}\right) \not \equiv 0(\bmod f)$. The proposition follows.

The following corollary is a reformulation of Proposition 3.3 .

Corollary 3.4. Let $g_{K}: Z_{K} \rightarrow \mathbb{P}_{K}^{1}$ be the $m$-cyclic cover of Lemma 3.2, Suppose that $p=\operatorname{char}(K)$ satisfies $p \geq m(f-3)$ and $p \equiv \alpha^{i}(\bmod m)$ for some $i \not \equiv 0$ $(\bmod f)$. Let $\pi_{K}: Y_{K} \rightarrow \mathbb{P}_{K}^{1}$ be the corresponding maximal meta-abelian cover. Then $\pi_{K}$ does not have a good degeneration.

\section{REFERENCES}

[1] I. I. Bouw. The p-rank of curves and covers of curves. In: Courbes semi-stables et groupes fondamental en géométrie algébrique (Luminy, 1998), Progress in Math. 187: 267-277, Birkhäuser, 2000. MR1768105(2001j:14042)

[2] I. I. Bouw. The p-rank of ramified covers of curves. Compositio Math., 126:295-322, 2001. MR1834740 (2002e:14045)

[3] A. Elkin. The rank of the Cartier operator on cyclic covers of the projective line. Preprint, arXiv:0708.0431.

[4] D. Harbater and K. Stevenson. Patching and thickening problems. J. Algebra, 212:272-304, 1999. MR1670658 (2000b:14004)

[5] B. Osserman. Linear series and existence of branched covers. Compositio Math., 144:89-106, 2008. MR2388557 (2009b:14056) 
[6] M. Romagny and S. Wewers. Hurwitz spaces. In: Groupes de Galois arithmétiques et différentiels. Séminaires et Congrès 13:313-341, Soc. Math. France, Paris, 2006. MR2316356 (2008e:14040)

[7] S. Wewers. Deformation of tame admissible covers of curves. In: Aspects of Galois theory (Gainesville, FL, 1996), London Math. Soc. Lecture Note Ser. 256:239-282, Cambridge Univ. Press, 1999. MR 1708609 (2001b:14048)

[8] S. Wewers and I. I. Bouw. Alternating groups as monodromy groups in positive characteristic. Pacific J. Math. 222:185-200, 2005. MR2200250(2006k:14050)

Institut für Reine Mathematik, Helmholtzstrasse 18, D-89069 Ulm, Germany

E-mail address: irene.bouw@uni-ulm.de 